\title{
Ideal Gas Laws
}

\section{Contents}

7.1 Two Ways of Knowing_Phenomenology and Microscopics ........... 334

7.1.1 Microscopic Models ........................................... 336

7.1.2 Phenomenological Models ................................... 338

7.1.3 Adiabatic Equation of State for an Ideal Gas ........................ 341

7.1.4 Adiabatic Temperature Change ............................... 342

$7.2 \quad$ Specific Heats of Ideal Gases ..................................... 343

7.2.1 Monatomic (Noble) Gases .......................................... 344

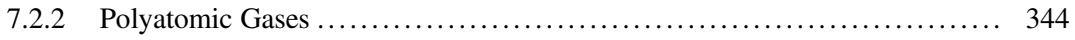

7.3 The Fundamental Equations of Hydrodynamics ................... 347

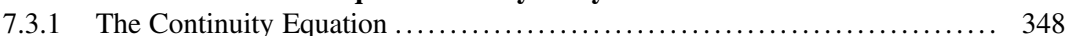

7.3.2 The Navier-Stokes (Euler) Equation ............................ 349

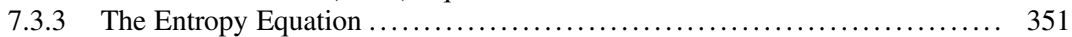

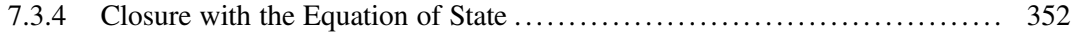

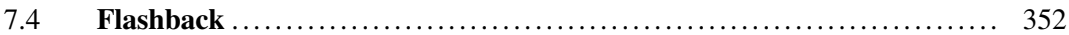

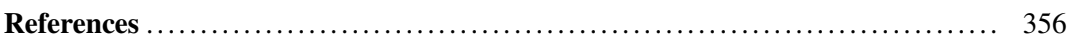

In Part II, our description of fluid behavior differs from our description of the dynamics of masses and springs, strings, bars, and two-dimensional vibrating surfaces. In Part I, it was reasonable to identify the coordinates of a specific point on such a vibrating system and write an equation for the time evolution of that point based on Newton's Second Law of Motion and some description of the appropriate elastic restoring force. With fluids, it is rare that we identify a "specific point" in the fluid and try to track the motion of that parcel of fluid. [1] With fluidic systems (gases, liquids, and plasmas), we generally adopt a different perspective, since it is inconvenient (and frequently impossible) to identify a specific "fluid particle" and track its flow under the influences of various forces and the constraints of boundaries.

Instead, we choose to identify a differential volume, $d V=d x d y d z$, specified in coordinates $(x, y$, and $z$ ) that are fixed in our laboratory frame of reference, while calculating the changes in the properties of the fluid within that differential volume (e.g., pressure, density, temperature, enthalpy, fluid velocity, mixture concentration, void fraction, dielectric polarization) as we keep track of the amount of fluid that enters or leaves that (fixed in space) differential volume. The fact that we no longer choose to identify individual fluid parcels requires the introduction of a mass conservation equation (also 
known as the continuity equation), in addition to our dynamical equation (Newton's Second Law) and our constitutive equation (Hooke's Law or some other equation of state).

When writing those three equations of fluid dynamics, which are expressed in coordinates specified in the laboratory frame of reference (rather than tracking a specific parcel of fluid), such equations are called Eulerian, after the great Swiss scientist and mathematician, Leonard Euler (1707-1783). In the case of sound waves in fluids, this is consistent with the way we would ordinarily make acoustic measurements in a fluid. Typically, a microphone or hydrophone or thermocouple or anemometer would be placed in a fixed position so we would like be able to interpret the pressures, velocities, and temperatures measured at that fixed location as the fluid moves to and fro.

There are special circumstances that allow the experimentalist to track a specific parcel of fluid by injecting a dye in a liquid or seeding a gas with small particles (e.g., smoke [1]) that move with the fluid. In such cases, that perspective is called Lagrangian, after Joseph-Louis Lagrange (1736-1813). ${ }^{1}$ With only very rare exception, our development and application of the hydrodynamic equations will take the Eulerian perspective.

The goal of Part II is to provide the tools to make predictions for fluid (liquid or gaseous) systems supporting wave-like disturbances that make such fluids depart from their state of static equilibrium. In this textbook, our focus will be on relatively small disturbances from equilibrium. For sound waves in air at atmospheric pressure, which are capable of creating permanent damage to your hearing with less than $15 \mathrm{~min}$ of exposure per day in a work environment [2], that acoustic pressure level $\left(115 \mathrm{~dB}_{\mathrm{SPL}}\right)$ corresponds to a peak excess pressure of only $16 \mathrm{~Pa}\left(1 \mathrm{~Pa}=1 \mathrm{~N} / \mathrm{m}^{2}\right)$. Since "standard" atmospheric pressure is $101,325 \mathrm{~Pa}$ [3], that potentially hazardous sound level corresponds to a relative deviation from equilibrium that is less than 160 parts per million (ppm) or $0.016 \%$.

\subsection{Two Ways of Knowing-Phenomenology and Microscopics}

"If, in some cataclysm, all of the scientific knowledge were to be destroyed, and only one sentence passed on to the next generations of creatures, what statement would contain the most information in the fewest words? I believe it is the atomic hypothesis; that all things are made of atoms."

R. P. Feynman (1918-1988) [4]

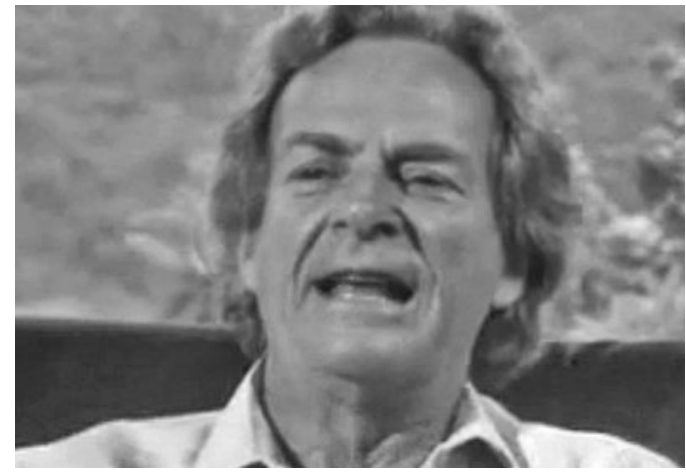

\footnotetext{
${ }^{1}$ Lagrange, who was of French/Italian descent (born Giuseppe Lodovico Lagrangia), was one of Euler's doctoral students. Euler recommended that Lagrange succeed him as the Director of Mathematics at the Prussian Academy of Sciences in Berlin.
} 
"Thermodynamics is the true testing ground of physical theory because its results are model independent. It is the only physical theory of universal content which I am convinced will never be overthrown, within the framework of applicability of its basic concepts."

A. Einstein (1879-1955)

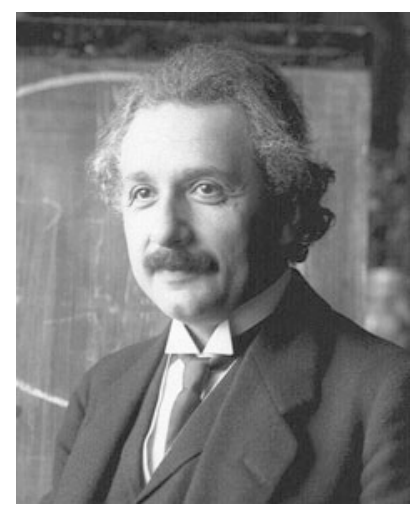

Scientists and engineers have a very rigorous definition of what constitutes "knowing." Fundamentally, scientific knowledge relies on testable hypotheses that can be verified (or falsified) experimentally and that have successfully withstood many such tests. If we say that we understand any given process or phenomenon, we mean that we can express that understanding using mathematics and we can make a quantitative calculation based on those mathematical expressions that will predict the outcome of a particular process or a specific situation, even if that device never existed or the process has never before been observed. Furthermore, our "understanding" permits us to estimate the uncertainty limits of those predictions (see Sect. 1.8). When that new process is executed, or that new device is created, the measured outcome or performance will have the predicted value within the predicted limits, if we have "understanding."

As mentioned in the Preface, the mathematical understanding must be supplemented by a "clear and intuitively satisfying narrative." When we have that, along with the predictive mathematics, we can usually intuit qualitative predictions before we make quantitative predictions; the qualitative and quantitative understandings provide a check on each other.

"It is always easier to do a calculation if you already know the result." I. Rudnick

Scientists have two fundamentally different ways of understanding natural phenomena on the scale sizes of human interest. These scales typically range from the "microscale," characterized by devices that have dimensions that are on the order of microns $\left(1\right.$ micron $\left.=1 \mu \mathrm{m}=10^{-6} \mathrm{~m}\right)$, to the "macroscale," such as the Earth's oceans and atmosphere, that have characteristic length scales in the thousands of kilometers $\left(10^{6} \mathrm{~m}\right)$. Although these concepts also apply on galactic scales, we usually call those people who are interested in galactic-scale phenomena cosmologists, astronomers, or astrophysicists, ${ }^{2}$ not acousticians.

\footnotetext{
${ }^{2}$ Acoustics is particularly important to cosmologists. For the first 300,000 years after the "Big Bang," all matter was ionized and therefore opaque to electromagnetic radiation. The only "channel" for wave propagation was acoustical. The residual cosmic background radiation is still evident with the "lumpiness" in the distribution of matter observed in the universe today that came from the time when those sound waves were "frozen" into the distribution of neutral matter: http://www.astro.ucla.edu/ wright/CMB-DT.html.
} 


\subsubsection{Microscopic Models}

One type of this rigorous "understanding" is expressed in the first quotation that started this section: "All matter is composed of atoms." We derive our understanding from that atomic perspective by combining the properties of those atoms with our knowledge of the interaction between those atoms. By averaging those interactions over large numbers of atoms, using the techniques of statistical mechanics, ${ }^{3}$ we can produce expressions for the behavior of bulk matter summarized by mathematical statements like the Ideal Gas Law, to relate stresses (pressure changes) to strains (density changes) as we did with the moduli of elasticity that characterized the elastic behavior of solids in Chap. 4. In this chapter, the kinetic theory of gases and the Equipartition Theorem (see Sect. 2.4.4) will be applied to the equation of state and to ball-and-stick models of molecules to provide some intuition about the acoustical properties and behaviors of gases.

The simplest application of this approach is the use of the kinetic theory of gases to derive the Ideal Gas Law. We start by considering a rectangular container with rigid walls that define a closed volume, $V=L_{x} L_{y} L_{z}$, containing $N$ atoms of a monatomic gas (e.g., helium or argon), as shown schematically in Fig. 7.1. We can regard those atoms as point particles, lacking an internal structure (unlike a molecule), each with mass, $m$, that can collide elastically ${ }^{4}$ with each other and scatter off of the rigid walls of the container.

If we focus our attention on a single atom that is moving with vector velocity, $\vec{v}$, we can calculate the momentum change for the $x$ component of momentum, $\Delta P_{x}=2 m v_{x}$, when that atom collides with the wall perpendicular to the $x$ axis and rebounds. Using Newton's Second Law of Motion, we know that the normal force on the wall, $F_{x}$, is proportional to the time rate of change of momentum. To calculate that rate, we need to know how many times that atom collides with the right wall per unit time. Since the projection of the atom's speed in the $x$ direction is $v_{x}$, it will collide with the right wall every $2 L_{x} / v_{x}$ seconds. The pressure exerted by that atom on that wall, $p_{x}$, is the ratio of that force, $F_{x}$, to the area of that wall, $A_{x}=L_{y} L_{z}$.

Fig. 7.1 Schematic representation of a gas of "point particles" confined to a volume, $V=L_{x} L_{y} L_{z}$. The velocity, $\vec{v}$, of one particle is indicated by the vector which has a projection in the $x$ direction of $v_{x}$

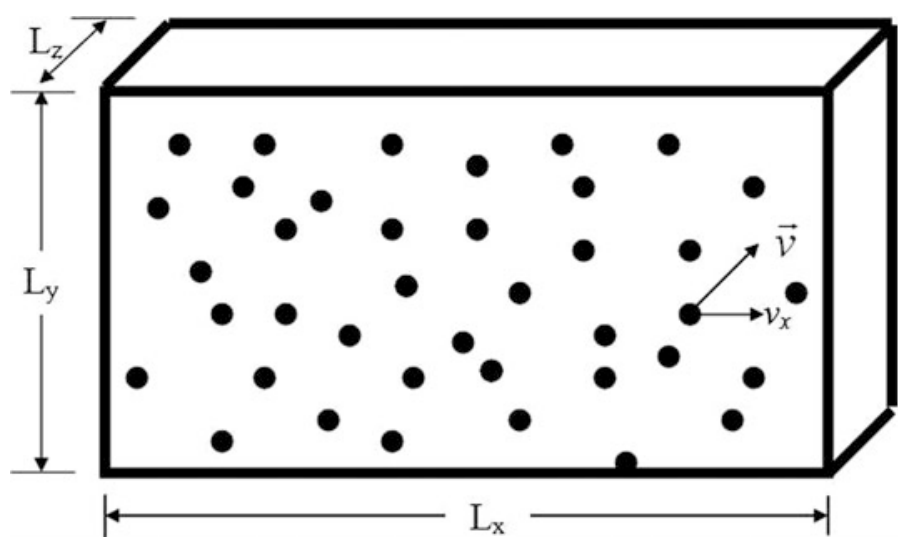

\footnotetext{
${ }^{3}$ The principles and methods of statistical mechanics are treated clearly and systematically at the advanced undergraduate level in the textbook by Fred Reif, Fundamentals of Statistical and Thermal Physics (McGraw-Hill, 1965); ISBN 07-051800-9.

${ }^{4}$ In an elastic collision, no energy is dissipated.
} 


$$
p_{x}=\frac{F_{x}}{A_{x}}=\frac{1}{L_{y} L_{z}} \frac{\Delta P_{x}}{\Delta t}=\frac{1}{L_{y} L_{z}} \frac{2 m v_{x}}{\left(2 L_{x} / v_{x}\right)}=\frac{m v_{x}^{2}}{V}
$$

If we recognize $m v_{x}^{2}$ as being twice the kinetic energy of that point particle associated with the $x$ direction degree of freedom of that particle's motion, then we can use the Equipartition Theorem (see Sect. 2.4.4) to relate the particle's average kinetic energy to the temperature of the gas in thermal equilibrium. The Equipartition Theorem states that on the average each available ${ }^{5}$ quadratic degree of freedom has an equal share of the system's energy and that share is equal to one-half of Boltzmann's constant, $k_{B} \equiv 1.380649 \times 10^{-23} \mathrm{~J} / \mathrm{K}=8.61733 \times 10^{-5} \mathrm{eV} / \mathrm{K}$ [5], times the absolute (kelvin) temperature, $T$, per degree of freedom.

For the atoms of a monatomic (noble) gas, which we have just treated as "point particles" in our calculation of the pressure on one wall of a three-dimensional box, each atom has three degrees of freedom; it can move right and left in the $x$ direction, up and down in the $y$ direction, and in and out of the page in the $z$ direction. Motion in each of the three orthogonal directions corresponds to three independent degrees of freedom. The average kinetic energy associated with each degree of freedom is "quadratic" in the sense that the kinetic energy depends upon the square of the velocity in each equivalent direction.

$$
\frac{1}{2} m\left\langle v_{x}^{2}\right\rangle=\frac{1}{2} m\left\langle v_{y}^{2}\right\rangle=\frac{1}{2} m\left\langle v_{z}^{2}\right\rangle=\frac{1}{2} k_{B} T
$$

The brackets indicate an average of the squared velocities where the average is taken over the entire $N$ particles within the volume.

In the laboratory, we measure the average pressure on the wall, but the "box" typically contains enormous numbers of atoms. We usually express the number of atoms or molecules in a more convenient form by introducing the number of atoms or molecules whose mass in grams is equal to the isotopic average of the atomic or molecular mass, $M$. The units of $M$ are grams/mole or kilograms/ mole. Using those definitions, $M=m N_{A}$. That number of atoms or molecules is known as Avogadro's number, $N_{A} \equiv 6.02214076 \times 10^{23} /$ mole. [5].

If the number of atoms in our box of volume, $V$, is $N$, then we can express that number as a fraction of Avogadro's number: $n=N / N_{A}$. Using Eq. (7.2) to substitute for $m<v_{x}{ }^{2}>$ in Eq. (7.1), we can express the pressure on the wall times the volume of the box in a form that is known as the Ideal Gas Law.

$$
p V=N m\left\langle v_{x}^{2}\right\rangle=\frac{N m}{3}\left\langle v^{2}\right\rangle=\frac{2 N}{3} \frac{1}{2} m\left\langle v^{2}\right\rangle=\frac{2 N}{3} \frac{3}{2} k_{B} T=n \Re T
$$

$\mathfrak{R} \equiv k_{B} N_{A} \equiv 8.314462 \mathrm{~J} /$ mole- $\mathrm{K}$ is the universal gas constant. ${ }^{6}$

The form of the Ideal Gas Law in Eq. (7.3), derived from the microscopic perspective, is expressed in that equation in terms of extensive variables. To maintain the pressure when we double the volume, we need to double the amount of gas. We may choose to express the Ideal Gas Law in terms of

\footnotetext{
${ }^{5}$ Whether or not a particular degree of freedom is "available" will be a consequence of quantum mechanics. Such quantum restrictions will be addressed specifically in Sect. 7.2.2.

${ }^{6}$ In a major redefinition of the International System of Units (SI, see Sect. 1.6), on 20 May 2019, the fundamental physical constants were assigned exact values, and other units, like the kilogram, were defined in terms of those physical constants. The universal gas constant was determined by acoustics experiments [L. Pitre, et al., Metrologia 54, 856-873 (2017)]. If we use the definition of standard conditions of temperature $\left(T=0{ }^{\circ} \mathrm{C}=273.15 \mathrm{~K}\right)$ and pressure $(P=1 \mathrm{~atm}=$ $101.325 \mathrm{kPa}$ ), then the volume of 1 mole of an ideal gas, under those conditions, is given by Eq. (7.3) as 22.414 liters $=$ $22.414 \times 10^{-3} \mathrm{~m}^{3}$.
} 
intensive variables that do not depend upon the size of the system: in this case, the volume of the container. We do this by introducing the mass density of the gas, $\rho=m N / V$, where $m N$ is the mass of the gas distributed uniformly within the volume, $V$.

$$
p=\frac{n \mathfrak{R} T}{V}=\frac{m N \mathfrak{R} T}{m N_{A} V}=\rho \frac{\mathfrak{R} T}{M}
$$

Again, $M=m N_{A}$, is the atomic or molecular mass of 1 mole of the ideal gas's constituents. In changing from $P_{x}$ to $p$, I have also invoked Pascal's law, which states that in a static fluid, pressure is isotropic. The pressure on the wall normal to the $x$ axis is the same as on all other walls. As stated by Blaise Pascal, in 1648: "In a body of equally dense fluid at rest, the pressure is the same for all points in the fluid so long as those points are at the same depth below the fluid's surface."7

Equation (7.4) is also commonly called the isothermal (constant temperature) equation of state for an ideal gas. It is an expression for $p$ in terms of $\rho: p=p(\rho, T)$, for an ideal gas at a constant absolute temperature, $T$. We will return to the microscopic view again as we explore the heat content of a fluid and investigate the consequences of treating polyatomic (molecular) gases instead of noble (monatomic) gases that will be represented as "point particles."

\title{
7.1.2 Phenomenological Models
}

\begin{abstract}
"Thermodynamics is a funny subject. The first time you go through it, you don't understand it at all. The second time you go through it, you think you understand it, except for one or two small points. The third time you go through it, you know you don't understand it, but by that time you are so used to it, it doesn't bother you anymore." (Arnold Sommerfeld [6])
\end{abstract}

A second approach to "knowing" is the phenomenological approach. We just completed a simple application of the microscopic (kinetic theory) perspective that was combined with the statistical mechanics (Equipartition) perspective to produce the Ideal Gas Law. Now we will take an approach that is not concerned about the particles that make up our fluid or their interactions. Instead, we start by asking how many macroscopic variables (e.g., pressure, temperature, fluid flow velocity, mixture concentration, void fraction, porosity, tortuosity, electrical charge density, electromagnetic field, gravitational field) are required to provide a complete description of our "system." We then have to write down the conservation laws (e.g., conservation of mass, conservation of charge, conservation of momentum) that "close" the system by invoking a number of conservation laws that equal the number of macroscopic variables. ${ }^{8}$

In this textbook, we will focus primarily on simple single-component fluids that are homogeneous (at equilibrium, their properties do not vary with position) and isotropic (at equilibrium, their properties do not vary with direction, for instance, the sound speed in all directions has the same value). There will be some useful and interesting cases where we will intentionally violate those assumptions. For example, we will study the sound speed in gas mixtures (like methane in air) and in

\footnotetext{
${ }^{7}$ We will deal with the part of Pascal's law that addresses "points are at the same depth below the fluid's surface" in Sect. 8.3.

${ }^{8}$ If the number of variables exceeds the number of conservation laws, the system is "underdetermined." If the number of conservation laws exceeds the number of variables, then the system is "overdetermined."
} 
bubbly fluids (hence, inhomogeneous fluids). We will also explore the Doppler shift in a fluid with a steady flow that makes the sound speed along the direction of flow different from the sound speed against the flow or perpendicular to the flow direction (hence, anisotropic).

For a single-component fluid at rest, only two variables are required to specify the state of the fluid. ${ }^{9}$ One variable will be mechanical, such as pressure, $p$, or density, $\rho$. The other variable will be thermal, such as thermodynamic (absolute) temperature, $T$, or entropy, $S$. We all have experience that gives us an intuitive understanding of $p, \rho$, and $T$, but $S$ can cause some discomfort because we do not have direct sensory experience with entropy. For our purposes, entropy is related to the heat content of our fluid. When the entropy of a closed system with spatially uniform temperature, $T$, is increased by an infinitesimal amount; $d S$, by means of the addition of an infinitesimal amount of heat; and $d Q$, to the system from its surroundings, these three quantities can be related.

$$
d S=\frac{d Q}{T}
$$

In acoustics, it is fairly common to consider systems where the heat that can enter or leave the system is negligibly small. ${ }^{10}$ Such systems are called adiabatic from the Greek: a, not; dia, through; and bainen, to go. Our adiabatic assumption leads to our first conservation equation.

$$
(d S)_{\text {adiabatic }}=0
$$

A more general treatment allows for the production of entropy by irreversible processes (e.g., heat conduction or viscous dissipation). In those cases, Eq. (7.5) and Eq. (7.6) become generalized to provide the Second Law of Thermodynamics, which says that the total entropy of any closed system can only increase with time.

$$
\dot{S}=\frac{d S}{d t} \geq 0
$$

To complete our description of this simple two-variable system, we need one more conservation law that is known as the First Law of Thermodynamics: energy can be converted from one type to another or moved from one place to another, but it cannot be created or destroyed.

$$
d U=d Q-d W
$$

The variable, $d U$, is the change of internal energy of the fluid. As before, $d Q$ is the small amount of heat added to the system, and $d W$ is the small amount of work done by the system. These sign conventions for $d W$ and $d Q$ reflect the historical emphasis on engines, not refrigerators, during the period when thermodynamics was being developed.

Since we will be restricting our attention to single-component fluids that are neither electrically charged nor magnetic, the only way we can do work on the fluid is mechanically, which is analogous to the work done by moving a particle against a force as expressed in Eq. (1.22) and Eq. (2.14).

\footnotetext{
${ }^{9}$ How do we know that two variables are enough? The best answer is that when we assume that two are enough, we get results that are consistent with experiment. Although I've heard arguments that the number of variables can be connected with "spontaneously broken symmetries," I do not understand (or necessarily believe) such arguments. Knowing the number of variables, a priori, is not necessary for a phenomenological theory. You can guess the number of variables, write the corresponding conservation laws, and then see if your theory explains your existing measurements and predicts some new behaviors that are testable..

${ }^{10}$ We'll calculate how small the heat leakage will be later in Sect. 9.3 when we address thermal conduction.
} 


$$
d W=p d V
$$

In this application, we can make the First Law of Thermodynamics more intuitive by considering a cylinder that is fitted with an idealized gas-tight frictionless piston. ${ }^{11}$

Using our definition of infinitesimal changes in entropy from Eq. (7.5) and of infinitesimal amounts of work in Eq. (7.9), we can rewrite the First Law of Thermodynamics in a form that will be useful for our calculation of the properties of an ideal gas.

$$
d Q=T d S=d U+p d V
$$

If we keep the piston's position fixed $(d V=0)$ and we add some thermal energy (heat), $d Q$, then the internal energy must increase by an amount $d U=d Q$, resulting in an increase in the temperature of the gas contained in the cylinder. If instead, we hold the pressure of the gas in the cylinder constant by letting the piston move while $d Q$ is added to the gas, then the piston will have to move outward, increasing the volume of the cylinder by $\mathrm{d} V$ and doing an amount of work, $d W=p d V$.

We are now in a position to determine how much heat is required to increase the temperature of an ideal gas by 1 degree kelvin if we combine our phenomenological result in Eq. (7.10) with the Ideal Gas Law in Eq. (7.3), derived from microscopic considerations. To simplify the mathematics, let us assume that our cylinder contains 1 mole of ideal gas ( $V_{o}=22.414$ liters at STP). We define the change in internal energy of the ideal gas per degree kelvin, when the volume is fixed, as the heat capacity at constant volume, $C_{V}$, also known as the isochoric heat capacity.

$$
C_{V} \equiv\left(\frac{\partial U}{\partial T}\right)_{V}=\left(\frac{\partial Q}{\partial T}\right)_{V}=T\left(\frac{\partial S}{\partial T}\right)_{V}
$$

Here we have to indicate that we are taking the derivative at constant volume, $V$, not at constant pressure, $p$.

Based on our representation of the idealized cylinder and piston in Fig. 7.2, it is clear that if we keep the pressure inside the cylinder constant, then when heat is added, the piston will have to move. Compared with a constant volume process, more heat will be required to cause the same change in temperature, since we are extracting work from the system as well as raising its temperature, hence, its internal energy.

Using the product rule for differentiation (see Sect. 1.1.2), we can calculate the differential form of the Ideal Gas Law in Eq. (7.3) for 1 mole of gas $(n=1$ mole).

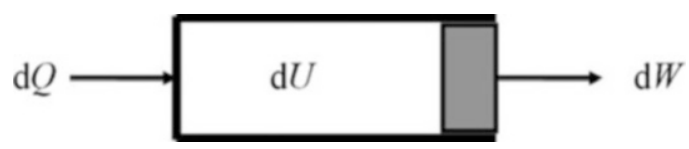

Fig. 7.2 An idealized frictionless piston sealing a cylinder containing a gas. The internal energy of the gas is increased by an amount, $\mathrm{d} U$, when an amount of heat, $\mathrm{d} Q$, is added or $\mathrm{d} U$ is decreased by an amount. $\mathrm{d} W=p$ d $V$, if work is extracted

\footnotetext{
${ }^{11}$ Although the "frictionless gas-tight piston" in a cylinder is convenient for pedagogical purposes, such frictionless gas pistons are approximated quite well by Airpot ${ }^{\circledR}$ Precision Air Dashpots. These have a very circular glass cylinder that is fitted to a graphite piston. Airpot Corp., Norwalk, CT 06852; www.airpot.com.
} 


$$
d(p V)=p d V+V d p=\mathfrak{R} d T
$$

Using the definition of isochoric heat capacity in Eq. (7.11) to express the differential heat input, $d Q$, in terms of $\mathrm{d} T$ and substituting $p d V$ from Eq. (7.12) into the First Law as expressed in Eq. (7.10), we can write the First Law in a way that will let us calculate the heat capacity if the addition of heat leads to both a temperature change, $d T$, and the production of work, $d W$, done by a volume change, $d V$, against a constant pressure, $p$.

$$
d Q=C_{V} d T+\mathfrak{R} d T-V d p=\left(C_{V}+\mathfrak{R}\right) d T-V d p
$$

By holding pressure constant $(d p=0)$, we can use Eq. (7.13) to express the heat capacity of an ideal gas at constant pressure, $C_{P}$, also called the isobaric heat capacity, in terms of the isochoric heat capacity, $C_{V}$, and the universal gas constant, $\mathfrak{R}$.

$$
C_{P}=\left(\frac{\partial Q}{\partial T}\right)_{p}=T\left(\frac{\partial S}{\partial T}\right)_{p}=C_{V}+\mathfrak{R}
$$

The fact that $C_{P}-C_{V}=\mathfrak{R}$ for 1 mole of gas is a general result for any ideal gas. It was derived by combining the (phenomenological) First Law of Thermodynamics with the (microscopic) Ideal Gas Law. However, Eq. (7.14) does not tell us how to calculate the value of $C_{V}$. To calculate $C_{V}$, we will have to return to our microscopic picture and to the Equipartition Theorem. Before doing so, it is convenient to use our phenomenological result to produce the equation of state for an ideal gas under adiabatic rather than isothermal conditions.

\subsubsection{Adiabatic Equation of State for an Ideal Gas}

We have defined a constant volume (isochoric) heat capacity, $C_{V}$, and a constant pressure (isobaric) heat capacity, $C_{P}$, for 1 mole of an ideal gas. We have also been able to relate the difference of those two heat capacities for 1 mole of any ideal gas: $C_{P}-C_{V}=\mathfrak{R}$. Of course, we could also define a generic heat capacity, $C$, that would allow both pressure and volume to vary simultaneously and in arbitrary proportions. Adiabatic and isothermal processes are idealized limits (like fixed and free boundary conditions for a string). There are entire ranges of processes that are intermediate.

The generic heat capacity, $C$, can be written in two ways. Using Eqs. (7.10) and (7.11), a generic heat capacity is related to volume changes.

$$
d Q=C d T=C_{V} d T+p d V
$$

Using Eqs. (7.13) and (7.14), a generic heat capacity, $C$, can also be written in terms of pressure changes.

$$
d Q=C d T=C_{P} d T-V d p
$$

Rearranging terms and dividing Eqs. (7.16) by (7.15) produces an equation for the ratio of the relative pressure change to the relative volume change.

$$
-\frac{V}{p} \frac{d p}{d V}=\frac{C_{p}-C}{C_{V}-C} \equiv \gamma^{\prime}
$$

The dimensionless constant, $\gamma^{\prime}$, has been introduced to make the result of the integration of Eq. (7.17) more compact. 


$$
\int \frac{d p}{p}=-\gamma^{\prime} \int \frac{d V}{V} \Rightarrow \ln p+\gamma^{\prime} \ln V=K \quad \Rightarrow \quad p V^{\gamma \prime}=\text { const }
$$

In this result, $K$ is just the integration constant, and the value of $\gamma$ ' will depend upon the process by which the pressure and volume are changed.

If we have an isothermal process, then $C=C_{i s o}=\infty$ in Eq. (7.15) or (7.16), because any amount of heat input, $d Q$, cannot cause the temperature to change. In that limit, substitution of $C_{i s o}=\infty$ into Eq. (7.17) requires that $\gamma^{\prime}=1$, and we recover the result of the isothermal equation of state in Eq. (7.3), where $p V=$ constant $=\mathfrak{R} T$ for a single mole of gas at constant (absolute) temperature, $T$.

For an adiabatic process, $d S=0$, as claimed in Eq. (7.6). Since $d T \neq 0$, we must require that $C=C_{\text {adiabat }}=0$, so when it is substituted into Eq. (7.15) or (7.16), $d Q=0$. Plugging $C_{\text {adiabat }}=0$ into Eq. (7.17) makes $\gamma^{\prime}=C_{P} / C_{V}$, therefore, the adiabatic equation of state for an ideal gas, also known as the Adiabatic Gas Law, takes its familiar form.

$$
p V^{\left(C_{P} / C_{V}\right)} \equiv p V^{\gamma}=p_{o} V_{o}^{\gamma}=\text { constant }
$$

The constant is determined when Eq. (7.19) is evaluated at some reference pressure, $p_{o}$, and reference volume, $V_{o}$. In the above expression, we have defined the ratio of the heat capacity at constant pressure to the heat capacity at constant volume to be $\gamma \equiv C_{P} / C_{V}$. The ratio, $\gamma$, is usually called the specific heat ratio or the polytropic coefficient because in a polytropic process, the heat capacities are taken to be independent of temperature. If we prefer to consider a unit mass of gas, then $\rho=m / V \propto$ $1 / \mathrm{V}$, so the adiabatic equation of state can be written in terms of the gas density.

$$
p \rho^{-\gamma}=p_{o} \rho_{o}^{-\gamma}=\mathrm{constant}
$$

Note that the constants in Eqs. (7.19) and (7.20) not only have different numerical values but also have different units.

\subsubsection{Adiabatic Temperature Change}

Of course, in an isothermal process, changes in pressure or density do not change the temperature of an ideal gas. For an adiabatic process, those pressure or density changes require that the temperature of the gas also change. We can calculate that temperature change, $\mathrm{d} T$, by substitution of the Ideal Gas Law of Eq. (7.3) into the adiabatic equation of state in Eq. (7.19).

$$
p V^{\gamma}=(n \Re T)^{\gamma} p^{1-\gamma} \Rightarrow T^{\gamma} p^{1-\gamma}=T_{o}^{\gamma} p_{o}^{\gamma-1}=\frac{p_{o} V_{o}^{\gamma}}{(n \mathfrak{R})^{\gamma}}=\text { constant }
$$

In Eq. (7.21), the constant is different from the constants in Eq. (7.19) or Eq. (7.20) because Eq. (7.21) has absorbed $(n \mathfrak{R})^{\gamma}$ into that constant. The above expression can be differentiated by ordinary means to derive an expression for $d T$ in terms of $d p$, but I would like to use the technique of logarithmic differentiation $^{12}$ to produce the same result because it is particularly convenient when dealing with power law expressions such as Eq. (7.21).

\footnotetext{
${ }^{12}$ This same calculation for the adiabatic temperature change in an ideal gas was done as an example of logarithmic differentiation in Sect. 1.1.3.
} 
We start by recalling the indefinite integral of $d x / x$ :

$$
\int \frac{d x}{x}=\ln x+C
$$

Here, $C$ is some constant of integration, not a heat capacity. By the Fundamental Theorem of Calculus (i.e., differentiation and integration are inverse processes), when we differentiate Eq. (7.22), we obtain an expression for the differential, $d x$. We can take the natural logarithm of the second form of Eq. (7.21).

$$
\gamma \ln T+(1-\gamma) \ln p=\ln (\text { constant })
$$

Differentiating Eq. (7.23) using the technique of Eq. (7.22) yields an expression for the relative change in absolute temperature, $d T / T$, and relative change in pressure, $d p / p$.

$$
\gamma \frac{d T}{T}=(\gamma-1) \frac{d p}{p}
$$

Since Eq. (7.24) was derived from the adiabatic equation of state in Eq. (7.19), we can rearrange terms to provide a useful expression for the change in temperature, $d T$, due to a change in pressure, $d p$, under adiabatic conditions.

$$
\left(\frac{\partial T}{\partial p}\right)_{S}=\frac{(\gamma-1)}{\gamma} \frac{T}{p}
$$

Notice that when $\gamma=1$, as it does for an isothermal process, $(\partial T / \partial p)_{S}=0$, consistent with the meaning of "isothermal."

As we will demonstrate later in this textbook, the propagation of sound in an ideal gas is very nearly an adiabatic process. If we reconsider the loud sound wave used as an example at the beginning of this chapter $\left(115 \mathrm{~dB}_{\mathrm{SPL}}\right)$, the magnitude of the peak pressure associated with that wave was $d p \equiv$ $p_{1}=16 \mathrm{~Pa}$. For air, $\gamma_{\text {air }} \cong 1.403$. If we assume the ambient temperature is $15^{\circ} \mathrm{C} \cong 288 \mathrm{~K}$, then the magnitude of the peak excess temperature amplitude due to a $115 \mathrm{~dB}_{\mathrm{SPL}}$ sound wave, $d T \equiv$ I $T_{1} \mathrm{I}=0.013 \mathrm{~K}=0.013^{\circ} \mathrm{C}^{13}$

\subsection{Specific Heats of Ideal Gases}

At this point, we have derived an expression for the difference between the constant pressure (isobaric) heat capacity per mole of an ideal gas, $C_{P}$, and the constant volume (isochoric) heat capacity per mole of an ideal gas, $C_{V}$. Our thermodynamic analysis showed that $C_{P}-C_{V}=\mathfrak{R}$, but we do not yet have an expression for either heat capacity and cannot therefore evaluate $\gamma$ for the adiabatic equation of state in Eq. (7.19) or for the expression in Eq. (7.25) that relates temperature changes to adiabatic pressure changes. To calculate $C_{V}$ for an ideal gas, we must return to our microscopic model and to the Equipartition Theorem of Eq. (7.2).

\footnotetext{
${ }^{13}$ Note that "degrees kelvin" can be abbreviated $[\mathrm{K}]$ without a degree sign but "degrees Celsius" requires the degree sign, $\left[{ }^{\circ} \mathrm{C}\right]$. This distinguishes it from the abbreviation for Coulomb, $[\mathrm{C}]=[\mathrm{A} \bullet \mathrm{s}]$, the SI unit of electrical charge.
} 


\subsubsection{Monatomic (Noble) Gases}

Our picture of noble gas atoms as "point particles" bouncing off each other and off rigid walls, but otherwise flying freely between collisions, implied that their only energy was kinetic. If we use the Equipartition Theorem, we can calculate the total average kinetic energy per particle by summing the average kinetic energy in all three degrees of freedom.

$$
\frac{1}{2} m\left\langle v^{2}\right\rangle=\frac{1}{2} m\left[\left\langle v_{x}^{2}\right\rangle+\left\langle v_{y}^{2}\right\rangle+\left\langle v_{z}^{2}\right\rangle\right]=\frac{3}{2} k_{B} T
$$

If we sum over a mole of particles, then we can calculate the internal (thermal) energy of 1 mole of this gas.

$$
U=\frac{3}{2} N_{A} k_{B} T=\frac{3}{2} \mathfrak{R T}
$$

From our definition of the isochoric heat capacity in Eq. (7.11), the heat capacity of 1 mole of a monatomic (noble) gas can be expressed in terms of the universal gas constant, $\mathfrak{R} \equiv k_{B} N_{A} \equiv 8.314462 \mathrm{~J} /$ mole-K.

$$
C_{V}=\left(\frac{\partial U}{\partial T}\right)_{V}=\frac{3}{2} \mathfrak{R}
$$

For noble (monatomic) ideal gases, the heat capacity at constant volume, $C_{V}=1.5 \Re \cong 12.472 \mathrm{~J} /$ mole-K. From Eq. (7.14), the isobaric heat capacity, $C_{P}=2.5 \Re \cong 20.786 \mathrm{~J} / \mathrm{mole}-\mathrm{K}$. Therefore, we can now calculate the ratio of specific heats for noble gases: $\gamma=C_{P} / C_{V}=5 / 3$. At sufficiently low pressures, this result is so precise that it was used to determine the universal gas constant by measuring sound speed in helium. [7]

In most cases, an intensive quantity, called the specific heat, is used to specify the heat capacity of a material, since the intensive quantity is independent of the size of the system. The most common specific heat is the heat capacity per unit mass. ${ }^{14}$ Using the convention of lowercase variables for intensive quantities (the most notable exception being the temperature, $T$ ), $c_{V}=1.5 \Re / M$ and $c_{P}=2.5$ $\mathfrak{R} / M$, where $M$ is the atomic mass. For example, helium has an atomic mass of $M_{H e}=4.0026 \mathrm{gm} / \mathrm{mole}$, so for helium at constant pressure, $c_{P} \cong 5.193 \mathrm{~J} / \mathrm{gm}-\mathrm{K}=5193 \mathrm{~J} / \mathrm{kg}-\mathrm{K}$.

\subsubsection{Polyatomic Gases}

If instead of a noble (monatomic) gas, we have a gas that is composed of stable (i.e., not chemically reacting) polyatomic molecules like $\mathrm{N}_{2}, \mathrm{O}_{2}, \mathrm{HCl}, \mathrm{H}_{2} \mathrm{O}, \mathrm{CO}_{2}, \mathrm{CH}_{4}$, etc., then we need to return to the Equipartition Theorem and calculate the number of "quadratic" degrees of freedom that are entitled to their "fair share" of the average thermal energy: (1/2) $k_{B} T$ per degree of freedom. Let's start with a simple symmetric diatomic molecule, like $\mathrm{N}_{2}$ or $\mathrm{O}_{2}$, shown schematically by the ball-and-stick model in Fig. 7.3.

In addition to the kinetic energy of the motion of the center of mass of the molecule that would contribute three "translational" degrees of freedom, as expressed in Eq. (7.26), this diatomic molecule

\footnotetext{
${ }^{14}$ In English units, the heat capacity is expressed in calories/gram where $1.0 \mathrm{cal}=4.184 \mathrm{~J}$. The nominal heat capacity of liquid water is $1.0 \mathrm{cal} / \mathrm{gram}$ because the calorie was originally defined as the amount of heat necessary to raise 1 gram of pure water from 19.5 to $20.5^{\circ} \mathrm{C}$.
} 
Fig. 7.3 Schematic representation of a diatomic molecule consisting of two identical atoms separated by an average distance $d$

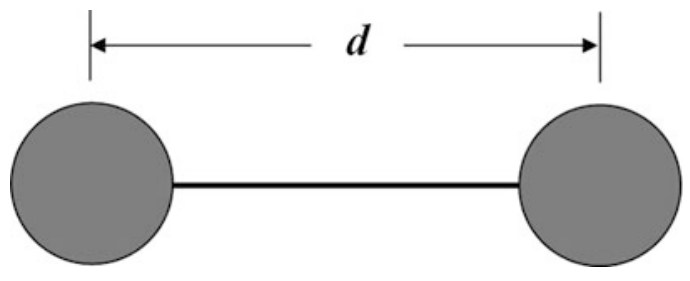

can rotate about its center of mass around two perpendicular axes (the third axis, along the line connecting the centers doesn't count for point masses) and could also vibrate along the line joining the two masses. Rotational kinetic energy, $(1 / 2) I \omega^{2}$, and the kinetic and potential energies of vibration, $K E=(1 / 2) \mu v^{2}$ and $P E=(1 / 2) \mathrm{K} x^{2}$, are also "quadratic" degrees of freedom (see Sect. 2.4.4), where $I$ is the moment of inertia for the pair of atoms about their center of mass, $\omega$ is the angular frequency of rotation, $\mathrm{K}$ is the effective spring constant for simple harmonic oscillation along the line connecting the two point masses (e.g., see Ch. 2, Prob. 13), and $\mu$ is the (effective) reduced mass of the pair (e.g., see Eq. 2.147) whose time-dependent separation is $d+x(t)$.

If we sum up the quadratic degrees of freedom, we get three "translational," plus two "rotational," plus two "vibrational" (i.e., one kinetic and one potential), for a total of seven "quadratic" degrees of freedom. If that were the case, then $C_{V}=(7 / 2) \Re$ and $C_{P}=(9 / 2) \Re$ for a diatomic gas. That is not the case for nitrogen at room temperature! The reason that result is incorrect can be traced to quantum mechanics. Remember, we are back to the "microscopic" model, and the world of atoms and molecules is governed by the laws of quantum mechanics, not classical mechanics.

In this case, it is useful to appreciate at least one specific instance where Planck's constant, ${ }^{15}$ $h \equiv 6.62607015 \times 10^{-34} \mathrm{~J}-\mathrm{s}=4.13566770 \times 10^{-15} \mathrm{eV}$-s, enters acoustics. In quantum mechanical systems, the spacing between energy levels is discrete, not continuous. We rarely see a direct manifestation of quantum effects in our daily life because Planck's quantum of action, $h$, is so small we can easily impart any amount of action (the product of the energy times the amount of time it is being applied) we choose to a macroscopic system. However, on the atomic scale, if there is insufficient energy to change the state of an atom or molecule by one quantized energy level, then no energy can be exchanged. ${ }^{16}$

How does all of this relate to the specific heat of a diatomic molecule? The energy levels for rotation, $E_{R}(j)$, and vibration, $E_{V}(n)$, are quantized with $j$ and $n$ being positive integers.

$$
E_{V}=(n+1 / 2) h v \quad \text { and } \quad E_{R}=\frac{j(j+1)}{2} \frac{h^{2}}{I}
$$

$I$ is the moment of inertia of the molecule and $\nu$ is the frequency of vibration.

Let's start by calculating the quantum of vibration, $h \nu$, for $\mathrm{N}_{2}$ as an example, then compare that energy to the average available kinetic energy per degree of freedom near room temperature $\left(T=290 \mathrm{~K} \cong 17^{\circ} \mathrm{C}\right):(1 / 2) k_{B} T=2 \times 10^{-21} \mathrm{~J}=0.0125 \mathrm{eV}$ per degree of freedom. (Electron volts tend to be a more convenient measure of the energy of individual atoms and molecules.)

\footnotetext{
${ }^{15}$ As of 20 May 2019, the value of Planck's constant has been assigned this "exact" value by SI System of Units. This definition led to the specification of the kilogram in terms of $h$, the speed of light, $c \equiv 299,792,458 \mathrm{~m} / \mathrm{s}$, and the length of the second based on the hyperfine transition frequency of cesium-133, $\Delta \nu_{C s} \equiv 9,192,531,770 \mathrm{~Hz}$. From those definitions, the kilogram is no longer based on the mass of a platinum-iridium cylinder near Paris [D. Newell, "A more fundamental International System of Units," Physics Today 67(7), 35-41 (2014)].

${ }^{16}$ This is quite fortunate for us, otherwise matter would not exist—electrons that orbit nuclei would radiate continuously and would spiral into their own nuclei.
} 
For $\mathrm{N}_{2}$, the vibrational frequency, measured by optical spectroscopy, is $\nu$ $\left(N_{2}\right)=8.2082 \times 10^{13} \mathrm{~Hz}=82,082 \mathrm{GHz}$. Such high frequencies are usually expressed by the corresponding wavelength of light, $\lambda_{E M}=c / f$, where $c \equiv 299,792,458 \mathrm{~m} / \mathrm{s}$, which is the speed of light in a vacuum. In this case, $\lambda_{E M}=3.65$ microns: a wavelength in the infrared portion of the electromagnetic spectrum. When multiplied by Planck's constant, $E_{V}\left(N_{2}\right)=h \nu=0.3395 \mathrm{eV}$. This corresponds to a vibrational temperature $T_{V}=E_{V} / k_{B} \cong 3940 \mathrm{~K}$. The probability of a molecule having sufficient energy to excite a vibrational mode is proportional to the Boltzmann factor, $P(E) .{ }^{17}$

$$
P(E)=e^{-E / k_{B} T}
$$

For our example using the vibrational frequency of $\mathrm{N}_{2}$, the probability of colliding with a molecule having sufficient kinetic energy to excite a vibrational mode, based on Eq. (7.30), is $P$ $\left(E_{V}\right)=1.25 \times 10^{-6}=1.25 \mathrm{ppm}$ at room temperature, about once chance in a million. For that reason, the vibrational degree of freedom does not contribute to the heat capacity of $\mathrm{N}_{2}$ at room temperatures.

For nitrogen, the rotational temperature, $T_{R}=E_{R} / k_{B}=2.86 \mathrm{~K}$, so by Eq. (7.30), the probability that an average room temperature atom will have sufficient energy to excite a rotational mode by an off-axis collision is nearly unity: $P\left(E_{R}\right)=0.99$. Only two rotational degrees of freedom exist for the diatomic molecule, since rotation about the axis joining the point particles does not correspond to actual rotation for the spherically symmetric end masses (since nearly all the mass is concentrated at the nucleus and rotations about that axis are indistinguishable). ${ }^{18}$

That leaves five accessible "quadratic" degrees of freedom for nitrogen near room temperature: three translational and two rotational. The specific heats for diatomic gases are therefore $c_{V}=(5 / 2) \mathfrak{R}$ and $c_{p}=(7 / 2) \mathfrak{R}$, so $\gamma=7 / 5=1.400$. The results for real diatomic gases are in quite good agreement with that model. For oxygen at $15{ }^{\circ} \mathrm{C}, \gamma=1.401$; nitrogen at $15{ }^{\circ} \mathrm{C}, \gamma=1.404$; and air at $0{ }^{\circ} \mathrm{C}$, $\gamma=1.403$. Of course, dry air is a mixture of about $78.1 \% \mathrm{~N}_{2}, 20.9 \% \mathrm{O}_{2}$ and $0.934 \% \mathrm{Ar}$, and about $415 \mathrm{ppm}$ of $\mathrm{CO}_{2}$ (and rising! ${ }^{19}$ ), so we expect the value of $\gamma_{\text {air }}$ to be a little larger than $7 / 5$ due to argon's contribution, which is monatomic and has $\gamma_{A r}=5 / 3$.

The graph in Fig. 7.4 demonstrates the effect of temperature on specific heat of $\mathrm{H}_{2}$. The rotational temperature of the hydrogen molecule, $T_{R}=85.6 \mathrm{~K}$, and the vibrational temperature, $T_{V} \cong 6100 \mathrm{~K}$. Below $T_{R}$, the molecule behaves as a monatomic gas and $C_{V}=(3 / 2) \Re$. Between 250 and $500 \mathrm{~K}$, the two rotational degrees of freedom can participate and $C_{V}=(5 / 2) \Re$. Above $500 \mathrm{~K}$, the two vibrational degrees of freedom are also becoming participatory. After dissociation, the number of the "particles" in the gas (now a plasma?) has doubled, so the heat capacity should be $2(3 / 2) \mathfrak{R}$ since there are 2 moles of monatomic hydrogen. ${ }^{20}$

\footnotetext{
${ }^{17}$ To be quantum mechanically correct, we should use the Planck distribution:

$P_{\text {Planck }}(E)=\left[e^{E / k_{B} T}-1\right]^{-1}$. Expansion of the exponential using a Taylor series makes it easy to see that for sufficiently high temperatures, $E \ll k_{B} T$, the Planck distribution reduces to the Boltzmann factor.

${ }^{18}$ Another way to appreciate the fact that rotation about the axis joining the two atoms is negligible is to remember that the moment of inertia is proportional to the mass times the square of the length of the "lever arm." For the two "dumbbell" rotational degrees of freedom, that lever arm is about half the atomic separation, $d$. For $\mathrm{N}_{2}, d \cong 1.0976 \times 10^{-10} \mathrm{~m} \cong 1.1$ $\AA$. The diameter of the nitrogen nucleus is about 1 femtometer $=1 \times 10^{-15} \mathrm{~m}=10^{-5} \AA$ (also called a Fermi), so the moment of inertia about the common axis is about $10^{10}$ times smaller than the moment of inertia for the "dumbbell" rotation.

${ }^{19}$ A. Gore, An Inconvenient Truth (Rodale Press, 2006); ISBN 1594865671

${ }^{20}$ The temperature dependence of the specific heat of hydrogen was measured nearly half a century before the "photoelectric effect" and the "ultraviolet catastrophe" were understood through the introduction of quantum mechanics and Planck's constant. With the benefit of hindsight, one can consider how the development of physics might have been altered if investigators had understood this macroscopic clue to the quantum character of the microscopic world.
} 


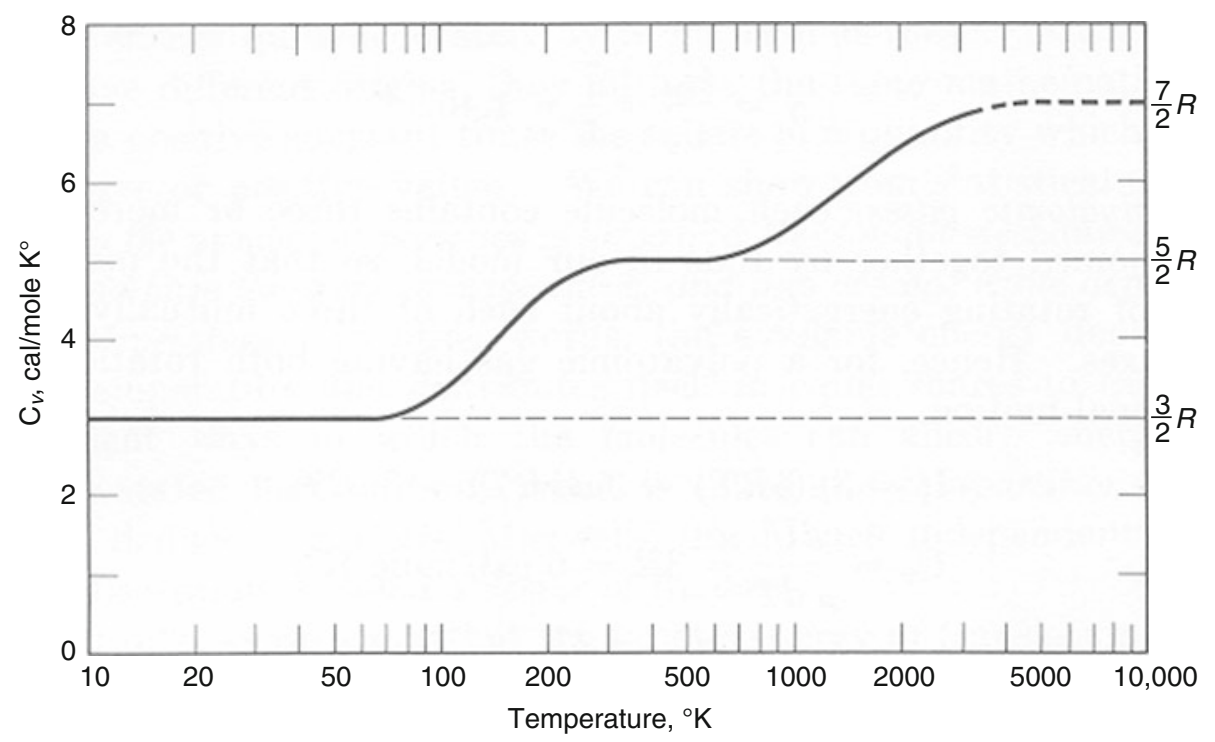

Fig. 7.4 Variation in the molar heat capacity at constant volume for $\mathrm{H}_{2}$ gas vs. temperature. [8]

We can continue to apply this simple view for more complex molecules. For molecular gases like $\mathrm{H}_{2} \mathrm{O}$ and $\mathrm{H}_{2} \mathrm{~S}$, there are three rotational degrees of freedom in addition to the three degrees of freedom associated with the motion of the molecule's center of mass; hence we expect $C_{V}=3 \Re$ and $C_{P}=4 \Re$, making $\gamma=4 / 3=1.33$. This is quite close for steam at $100{ }^{\circ} \mathrm{C}(\gamma=1.324)$ and for $\mathrm{H}_{2} \mathrm{~S}$ at $15{ }^{\circ} \mathrm{C}$ $(\gamma=1.32)$. The situation for $\mathrm{CO}_{2}$ at $15{ }^{\circ} \mathrm{C}(\gamma=1.304)$ is a bit more complicated. Based on this analysis, we expect the more complex molecular gases to have still smaller values of $\gamma$. In any case, the polytropic coefficient for any ideal gas is bounded: $1<\gamma \leq 5 / 3$.

As we will see later in Chap. 14, when we investigate attenuation mechanisms in air and sea water, the heat capacity is not only temperature-dependent, but it is also time-dependent. For example, consider a loudspeaker in contact with air. The impact of the speaker cone on the air molecules transfers only translational kinetic energy. The molecules then have to make several collisions (about five, on average) to distribute the additional energy equitably between translational and rotational modes, as dictated by the Equipartition Theorem, which is a statement about equilibrium. It takes a non-zero amount of time to re-establish that equilibrium and the "relaxation time" (see Sect. 4.4.1) for that process, $\tau_{R}$, can introduce phase shifts and hence dissipation. These relaxation effects are usually lumped into a term known as the "bulk viscosity." 21

\subsection{The Fundamental Equations of Hydrodynamics}

\footnotetext{
"An acoustician is merely a timid hydrodynamicist." (A. Larraza)
}

\footnotetext{
${ }^{21}$ The "bulk viscosity" or "second viscosity" is a correction for the fact that there is a sixth "relaxing" variable in a system of phenomenological equations based on only five variables (see Sect. 14.5). The introduction of this "relaxation time," $\tau_{R}$, which is responsible for the delayed equilibration between internal degrees of freedom, is reflected in a timedependent specific heat, introduced in a rigorous manner in L. D. Landau and E. M. Lifshitz, Fluid Mechanics (Pergamon, 1959), §78, entitled "Second Viscosity."
} 
The previous discussion has introduced the concept of microscopic and phenomenological models by calculating some acoustically useful properties of ideal gases. Equilibrium thermodynamics was our first example of a phenomenological theory. Two variables were required to describe the static, singlecomponent, homogeneous, isotropic, electromagnetically inert fluid. Two conservation laws (entropy and energy) were used to "close" that system.

For acoustics, static equilibrium is an unacceptable restriction; sound waves make fluids move! To produce a phenomenological theory that will incorporate acoustical effects, we need to introduce another three variables; the three components of the velocity vector.

$$
\vec{v}=v_{x} \widehat{e}_{x}+v_{y} \widehat{e}_{y}+v_{z} \widehat{e}_{z}
$$

As before, $v_{x}$ is the $x$ component of velocity, and $\widehat{e}_{x}$ has been introduced as the unit vector in the $x$ direction.

To close this chapter, I will list the conservation equations that close the five-variable system describing the hydrodynamic behavior of a single-component, electrically neutral, nonmagnetic, homogeneous, isotropic fluid. The goal of the next two chapters is to provide an understanding of these equations at a level that will empower you to apply them to acoustical problems and be able to modify them when necessary for more complicated situations. A good reference for such hydrodynamic equations is Fluid Mechanics, by Landau and Lifshitz. [9].

\subsubsection{The Continuity Equation}

In the introduction to this chapter, I pointed out that our Eulerian perspective for expression of the equations governing fluids was based on a preference for expressing fluid properties (i.e., pressure, density, temperature, entropy, and fluid velocity) in a fixed frame of reference defined by the laboratory and not on the fluid parcels themselves; the fluid moves in and out of our differential Eulerian volumes, $d V=d x d y d z$. In Part I of this textbook, all of the "particles" had equilibrium positions, and we could apply an equation of state (like Hooke's law) and Newton's Second Law of Motion to derive the vibrations of masses on springs, strings, bars, membranes, and plates.

Since the fluid particles are not tied to the laboratory coordinate system, we need another equation, in addition to the equation of state and dynamic equation, to keep track of the fluid in our chosen Eulerian perspective. That third equation ensures conservation of mass and is also known as the continuity equation. It can be written in vector form.

$$
\frac{\partial \rho}{\partial t}+\nabla \cdot(\rho \vec{v})=0
$$

Equation (7.32) has the form of a "conservation equation." It is the sum of the time derivative of a density, $\rho$ (in this case the mass density with units of $\mathrm{kg} / \mathrm{m}^{3}$ ), plus the divergence of a flux. The flux is the mass flux, $\vec{J}=\rho \vec{v}=J_{x} \widehat{e}_{x}+J_{y} \widehat{e}_{y}+J_{z} \widehat{e}_{z}$, that represents the amount of mass that flows through a unit area in unit time. The divergence operator converts a vector, such as the mass flux in Eq. (7.32), to a scalar. It can be written in the coordinate system that is appropriate for the description of the problem of interest. The simplest version of the divergence operator $(\nabla \cdot)$ can be written in Cartesian coordinates.

$$
(\nabla \cdot) \vec{J}=\frac{\partial}{\partial x} J_{x}+\frac{\partial}{\partial y} J_{y}+\frac{\partial}{\partial z} J_{z}
$$


The divergence operator has the units of (length) ${ }^{-1}$ in any coordinate system, so the expression for conservation of mass in Eq. (7.32) is dimensionally homogeneous, as it must be. If there were sources or sinks within the fluid, or if there was a time-dependent (i.e., moving) boundary, then the right-hand side of Eq. (7.32) would no longer be zero. When radiation of sound from vibrating objects is considered in Chap. 12, loudspeakers or other sound sources would provide such "source terms."

\subsubsection{The Navier-Stokes (Euler) Equation}

Newton's Second Law of Motion, $m \ddot{x}=F_{n e t}$, takes a different form when expressed in Eulerian coordinates, requiring a redefinition of the acceleration of a "fluid parcel" that is within a differential volume that is fixed in the laboratory coordinate system, $d V=d x d y d z$. It must include both the acceleration of the fluid within the parcel, $\partial \vec{v} / \partial t$, as well as the acceleration of the fluid that enters and leaves the parcel by convection, $(\vec{v} \bullet \vec{\nabla}) \vec{v}$.

$$
\rho\left[\frac{\partial \vec{v}}{\partial t}+(\vec{v} \cdot \vec{\nabla}) \vec{v}\right]=-\vec{\nabla} p+\mu \nabla^{2} \vec{v}+\rho \vec{g}
$$

This version (known as the Navier-Stokes equation) assumes that the shear viscosity, $\mu$, is not a function of either velocity or position and that the acceleration due to gravity, $\vec{g}$, is not a function of position or time.

The Laplacian operator, $\nabla^{2}$, is a scalar operator that can be expressed most simply in Cartesian coordinates.

$$
\nabla^{2}=\frac{\partial^{2}}{\partial x^{2}}+\frac{\partial^{2}}{\partial y^{2}}+\frac{\partial^{2}}{\partial z^{2}}
$$

When it is applied to a vector, it generates another vector.

$$
\nabla^{2} \vec{v}=\widehat{e}_{x} \frac{\partial^{2} v_{x}}{\partial x^{2}}+\widehat{e}_{y} \frac{\partial^{2} v_{y}}{\partial y^{2}}+\widehat{e}_{z} \frac{\partial^{2} v_{z}}{\partial z^{2}}
$$

The gradient operator, $\vec{\nabla}$, is a vector operator that can also be expressed in Cartesian coordinates.

$$
\vec{\nabla}=\widehat{e}_{x} \frac{\partial}{\partial x}+\widehat{e}_{y} \frac{\partial}{\partial y}+\widehat{e}_{z} \frac{\partial}{\partial z}
$$

When $\vec{\nabla}$ operates on a scalar, like $p$, it creates a vector.

$$
\vec{\nabla} p=\widehat{e}_{x} \frac{\partial p}{\partial x}+\widehat{e}_{y} \frac{\partial p}{\partial y}+\widehat{e}_{z} \frac{\partial p}{\partial z}
$$

When $\vec{\nabla}$ operates on a vector, like $\vec{v}$, it creates a 3 x 3 tensor. [10] 


$$
\vec{\nabla} \vec{v}=\left|\begin{array}{lll}
\widehat{e}_{x} \frac{\partial v_{x}}{\partial x} & \widehat{e}_{y} \frac{\partial v_{x}}{\partial y} & \widehat{e}_{z} \frac{\partial v_{x}}{\partial z} \\
\widehat{e}_{x} \frac{\partial v_{y}}{\partial x} & \widehat{e}_{y} \frac{\partial v_{y}}{\partial y} & \widehat{e}_{z} \frac{\partial v_{y}}{\partial z} \\
\widehat{e}_{x} \frac{\partial v_{z}}{\partial x} & \widehat{e}_{y} \frac{\partial v_{z}}{\partial y} & \widehat{e}_{z} \frac{\partial v_{z}}{\partial z}
\end{array}\right|
$$

The dot product of the velocity vector, $\vec{v}$, and the tensor, $\vec{\nabla} \vec{v}$, produces a vector.

$$
(\vec{v} \cdot \vec{\nabla}) \vec{v}=\widehat{e}_{x} v_{x} \frac{\partial v_{x}}{\partial x}+\widehat{e}_{y} v_{y} \frac{\partial v_{y}}{\partial y}+\widehat{e}_{z} v_{z} \frac{\partial v_{z}}{\partial z}
$$

Equation (7.34) is a vector equation, so it is actually three separate equations for the three components of velocity. In Cartesian coordinates, those components would be $v_{x}, v_{y}$, and $v_{z}$. In cylindrical coordinates, they are $v_{r}, v_{\theta}$, and $v_{z}$. In spherical coordinates, they are $v_{r}, v_{\theta}$, and $v_{\phi}$. To demonstrate that Eq. (7.34) is not as intimidating as the vector operations above might suggest, the following is the $x$ component of that equation.

$$
\rho\left[\frac{\partial v_{x}}{\partial t}+v_{x} \frac{\partial v_{x}}{\partial x}\right]=-\frac{\partial p}{\partial x}+\mu\left[\frac{\partial^{2} v_{x}}{\partial x^{2}}+\frac{\partial^{2} v_{x}}{\partial y^{2}}+\frac{\partial^{2} v_{x}}{\partial z^{2}}\right]+\rho g_{x}
$$

The last term on the right-hand side uses $g_{x}$ to indicate the $x$ component of the gravitational acceleration.

The fluid density times the quantity in square brackets is the one-dimensional definition of the time rate of change of the momentum in an Eulerian coordinate system, as will be justified in the next chapter. It states that the mass density times the fluid acceleration plus the flow of momentum is equal to the net force on a "fluid element." In this case, the forces included in Eq. (7.34) and its $x$ component in Eq. (7.41) are those due to pressure gradients, $\vec{\nabla} p$; shear stresses, $\mu \nabla^{2} \vec{v}$; and the acceleration due to gravity, $\rho \vec{g}$. The quantity, $\mu$, is known as the shear viscosity and it has MKS units of [Pa-s]. In the form shown in Eq. (7.34), Newton's Second Law of Motion is known as the Navier-Stokes Equation. ${ }^{22}$ If the only forcing term is the pressure gradient, the equation is known as the Euler Equation.

$$
\rho\left[\frac{\partial \vec{v}}{\partial t}+(\vec{v} \cdot \vec{\nabla}) \vec{v}\right]=-\vec{\nabla} p
$$

Later in this textbook, other terms will be added to the right-hand side of Eq. (7.34), such as flow resistance for porous materials like those used in acoustical ceiling tiles, or "bulk viscosity" to account for relaxation time effects. ${ }^{21}$ On the other hand, we will usually ignore terms like $\rho \vec{g}$ where such terms might have a negligible influence. ${ }^{23}$

As will be demonstrated in Sect. 10.5, energy conservation is contained within the combination of the continuity Eq. (7.32) and the Euler Eq. (7.42).

\footnotetext{
${ }^{22}$ The version of the Navier-Stokes equation in Eq. (7.34) does not include the "bulk viscosity" mentioned in footnote 21. It will be added later since it has significant impact in the attenuation of sound as discussed in Sect. 14.5.

${ }^{23}$ The $\rho \vec{g}$ term will be important for waves on the free surface of water (e.g., tsunamis, surf) or for acoustic oscillations of planetary atmospheres that are generated by seismic events, volcanic explosions, meteors, etc.
} 


\subsubsection{The Entropy Equation}

Entropy production was already mentioned in Eq. (7.7). An equation for entropy production can also be written in Eulerian coordinates.

$$
\rho T\left[\frac{\partial s}{\partial t}+(\vec{v} \cdot \vec{\nabla}) s\right]=\vec{\nabla} \cdot \kappa \vec{\nabla} T+(\stackrel{\leftrightarrow}{\sigma} \cdot \vec{\nabla}) \cdot \vec{v}
$$

Again, the term in square brackets indicates that the (scalar) entropy per unit mass, $s$, can change within the Eulerian "fluid parcel" with respect to time, $\partial s / \partial t$, and can change if entropy is transported (convected) into or out of the parcel, $(\vec{v} \bullet \vec{\nabla}) s$. It is also worthwhile to recognize that Eq. (7.43) is a scalar (not vector) equation. The gradient operator, $\vec{\nabla}$, converts $s$ into a vector.

$$
\vec{\nabla} s=\widehat{e}_{x} \frac{\partial s}{\partial x}+\widehat{e}_{y} \frac{\partial s}{\partial y}+\widehat{e}_{z} \frac{\partial s}{\partial z}
$$

The dot product with $\vec{v}$ then reduces this term back to a scalar.

$$
\vec{v} \cdot \vec{\nabla} s=v_{x} \frac{\partial s}{\partial x}+v_{y} \frac{\partial s}{\partial y}+v_{z} \frac{\partial s}{\partial z}
$$

This is a bit more complicated than the version of entropy conservation we used for our discussion of ideal gases, expressed in Eq. (7.6), because it allows for two sources of entropy generation: the first, $\nabla \cdot \kappa \vec{\nabla} T$, allows entropy (heat) transport by thermal conduction, where $\kappa[\mathrm{W} / \mathrm{m}-\mathrm{K}]$ is the fluid's thermal conductivity. The second allows entropy to be generated by viscous effects defined by a nine-component viscous stress tensor, $\stackrel{\leftrightarrow}{\sigma}$. The rate of specific entropy generation, $\dot{s}_{g e n}$, is provided by Swift. [11]

$$
\dot{s}_{\text {gen }}=\frac{\kappa|\nabla T|^{2}}{\rho T^{2}}+\frac{1}{\rho T}(\stackrel{\leftrightarrow}{\sigma} \cdot \vec{\nabla}) \cdot \vec{v}>0
$$

Equation (7.49) shows that $\stackrel{\leftrightarrow}{\sigma}$ is proportional to velocity so the last term in Eq. (7.46) is proportional to $|\vec{v}|^{2}$ and is always positive, as is the term involving $|\vec{\nabla} T|^{2}$, so $\dot{s}_{g e n}>0$, as required by the Second Law of Thermodynamics.

As in the case illustrated by Eq. (7.44), a vector is created by taking the gradient of the (scalar) temperature.

$$
\vec{\nabla} T=\widehat{e}_{x} \frac{\partial T}{\partial x}+\widehat{e}_{y} \frac{\partial T}{\partial y}+\widehat{e}_{z} \frac{\partial T}{\partial z}
$$

The dot product of the gradient operator, $\vec{\nabla}$, and $\kappa \vec{\nabla} T$ again produces a scalar.

$$
\vec{\nabla} \cdot \kappa \vec{\nabla} T=\frac{\partial}{\partial x}\left(\kappa \frac{\partial T}{\partial x}\right)+\frac{\partial}{\partial y}\left(\kappa \frac{\partial T}{\partial y}\right)+\frac{\partial}{\partial z}\left(\kappa \frac{\partial T}{\partial z}\right)
$$

Of course, if $\kappa$ is not a function of position, it can be taken outside the derivatives.

The last term in Eq. (7.43) accounts for the entropy generated by dissipation in viscous flow that is expressed by the viscous stress tensor, $\stackrel{\leftrightarrow}{\sigma}$. Neglecting "bulk viscosity,"21,22 this nine-component tensor can also be expressed in Cartesian coordinates, where we have assumed that the shear viscosity is neither a function of position or velocity. 


$$
\stackrel{\leftrightarrow}{\sigma}=\mu\left|\begin{array}{ccc}
-\widehat{e}_{x}\left(\frac{4}{3} \frac{\partial v_{x}}{\partial x}-\frac{2}{3} \frac{\partial v_{y}}{\partial y}-\frac{2}{3} \frac{\partial v_{z}}{\partial z}\right) & \widehat{e}_{y}\left(\frac{\partial v_{x}}{\partial y}+\frac{\partial v_{y}}{\partial x}\right) & \widehat{e}_{z}\left(\frac{\partial v_{x}}{\partial z}+\frac{\partial v_{z}}{\partial x}\right) \\
\widehat{e}_{x}\left(\frac{\partial v_{y}}{\partial x}+\frac{\partial v_{x}}{\partial y}\right) & -\widehat{e}_{y}\left(\frac{4}{3} \frac{\partial v_{y}}{\partial y}-\frac{2}{3} \frac{\partial v_{x}}{\partial x}-\frac{2}{3} \frac{\partial v_{z}}{\partial z}\right) & \widehat{e}_{z}\left(\frac{\partial v_{y}}{\partial z}+\frac{\partial v_{z}}{\partial y}\right) \\
\widehat{e}_{x}\left(\frac{\partial v_{z}}{\partial x}+\frac{\partial v_{x}}{\partial z}\right) & \widehat{e}_{y}\left(\frac{\partial v_{z}}{\partial y}+\frac{\partial v_{y}}{\partial z}\right) & -\widehat{e}_{z}\left(\frac{4}{3} \frac{\partial v_{z}}{\partial z}-\frac{2}{3} \frac{\partial v_{x}}{\partial x}-\frac{2}{3} \frac{\partial v_{y}}{\partial y}\right)
\end{array}\right|
$$

The dot product of the stress tensor with the gradient operator creates a vector, and the dot product of that vector with the velocity is again a scalar.

\subsubsection{Closure with the Equation of State}

We now have five hydrodynamic equations but now have gone from five variables to seven because we introduced density, $\rho$, as a variable in the Navier-Stokes equation written in Eq. (7.34), and specific entropy (per unit mass), $s$, that was introduced by the entropy equation written in Eq. (7.43). This underdetermined problem can be "closed" by introducing two equations of state. The first relates mass density, $\rho$, to pressure, $p$, and absolute temperature, $T$.

$$
\rho=\rho(p, T)
$$

We have already introduced an equation of state for an ideal gas that undergoes processes that are either isothermal in Eq. (7.4) or adiabatic in Eq. (7.20). We can do the same for any fluid, although the equations of state can become rather complicated.

Since we allowed for entropy generation in Eq. (7.46), we also need to be able to relate entropy and temperature.

$$
s=s(p, T)
$$

One possible relationship between entropy and temperature was established previously with the introduction of the isochoric heat capacity in Eq. (7.11), so $d s=c_{V} T d T$, and the isobaric heat capacity in Eq. (7.14), so $d s=c_{P} T d T$. In effect, we bring ourselves back to the assumption of five variables by recognizing that $\rho$ and $s$ are not independent variables but will be unique functions of $p$ and $T$ for any substance through which sound propagates.

It is important to recognize the difference between the three conservation equations and the equations of state. The form of the conservation laws are fluid independent, as long as the fluids obey our initial assumptions (e.g., single-component, electrically neutral and insulating, nonmagnetic, homogeneous and isotropic). The equations of state may differ for different fluids even though those fluids obey our initial assumptions.

Now armed with the Eulerian equations of hydrodynamics and the Ideal Gas Laws, we are ready to start exploring acoustics in fluid media. We will initiate this exploration in the next chapter by applying those equations to the simplest cases.

\subsection{Flashback}

In this chapter, two complementary descriptions of matter were introduced: microscopic and phenomenological. The microscopic (statistical mechanical) description led us to the Ideal Gas Law, and the 
macroscopic (thermodynamic) description made it possible for us to relate the isobaric (constant pressure) and isochoric (constant volume) specific heats of an ideal gas.

To determine values for the specific heats, and their ratio (the polytropic coefficient), it was necessary to return to the microscopic viewpoint and consider the internal degrees of freedom of the particles. The "availability" of those internal degrees of freedom to influence the acoustical and thermodynamic properties of the ideal gas turned out to require the introduction of some elementary concepts from quantum mechanics.

The final topic in this chapter was the introduction of the equations of hydrodynamics and the equations of state. The generality of those equations makes them difficult to comprehend, although their algebraic structure suggested that they formed a "closed description" for a single-component, homogeneous, isotropic fluid, since the system provided five conservation laws for five phenomenological variables: a mechanical property of the fluid, $p$ (or $\rho$ ), a thermal property of the fluid, $T$ (or $s$ ), and three components of the fluid's velocity, expressed in Cartesian coordinates as $v_{x}, v_{y}$, and $v_{z}$. In the next chapter, their form will be examined, and they will be applied to hydrostatics and simple acoustical components that are small compared to the wavelength of sound.

Talk Like an Acoustician

$\begin{array}{ll}\text { Ideal Gas Law } & \text { Heat capacity at constant volume } \\ \text { Kinetic theory of gases } & \text { Isochoric heat capacity } \\ \text { Inert gas } & \text { Heat capacity at constant pressure } \\ \text { Point particles } & \text { Isobaric heat capacity } \\ \text { Equipartition Theorem } & \text { Adiabatic gas law } \\ \text { Quadratic degree of freedom } & \text { Adiabatic equation of state } \\ \text { Boltzmann's constant } & \text { Specific heat ratio } \\ \text { Avogadro's number } & \text { Logarithmic differentiation } \\ \text { Universal Gas Constant } & \text { Monoatomic (noble) gas } \\ \text { Extensive variables } & \text { Polyatomic molecules } \\ \text { Intensive variables } & \text { Planck's constant } \\ \text { Pascal's law } & \text { Boltzmann factor } \\ \text { Isothermal equation of state } & \text { Planck distribution } \\ \text { Macroscopic variables } & \text { Conservation of mass } \\ \text { Conservation laws } & \text { Continuity equation } \\ \text { Homogeneous fluid } & \text { Navier-Stokes equation } \\ \text { Isotropic fluid } & \text { Euler equation } \\ \text { Entropy } & \text { Shear viscosity } \\ \text { Adiabatic } & \text { Thermal conductivity } \\ \text { Second Law of Thermodynamics } & \text { Viscous stress tensor } \\ \text { First Law of Thermodynamics } & \end{array}$

\section{Exercises}

1. A billion of 'em. One mole ideal gas (e.g., air) at conditions of standard temperature $(273.15 \mathrm{~K})$ and pressure $(101,325 \mathrm{~Pa})$ occupies a volume of 22.414 liters/mole. The wavelength of sound, $\lambda$, is given by the ratio of the speed of sound, $c$, to the frequency, $f: \lambda=c / f$. Avogadro's number, $N_{A} \equiv 6.02214076 \times 10^{23} \mathrm{~mol}^{-1}$.

The speed of sound in air under the same conditions of temperature and pressure is $c=331.65 \mathrm{~m} /$ sec. If we insist that a cubic Eulerian parcel of air contains an average of a billion gas particles, what is the highest frequency of sound in air that would correspond to the wavelength of sound being ten times longer than an edge of the Eulerian parcel? 

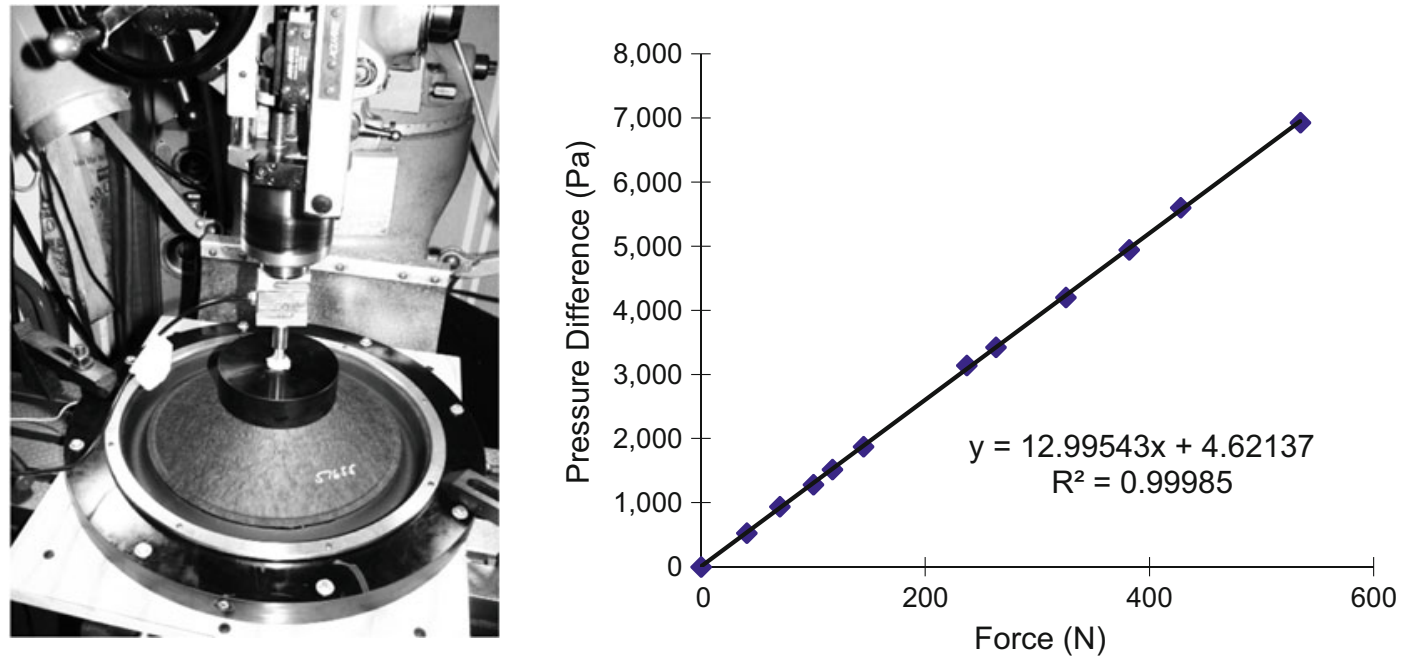

Fig. 7.5 (Left) Photograph of an apparatus that can determine the force on a loudspeaker cone and surround (see Fig. 2.17) due to a pressure difference across the cone. (Right) Graph of the pressure difference vs. measured force on the cone
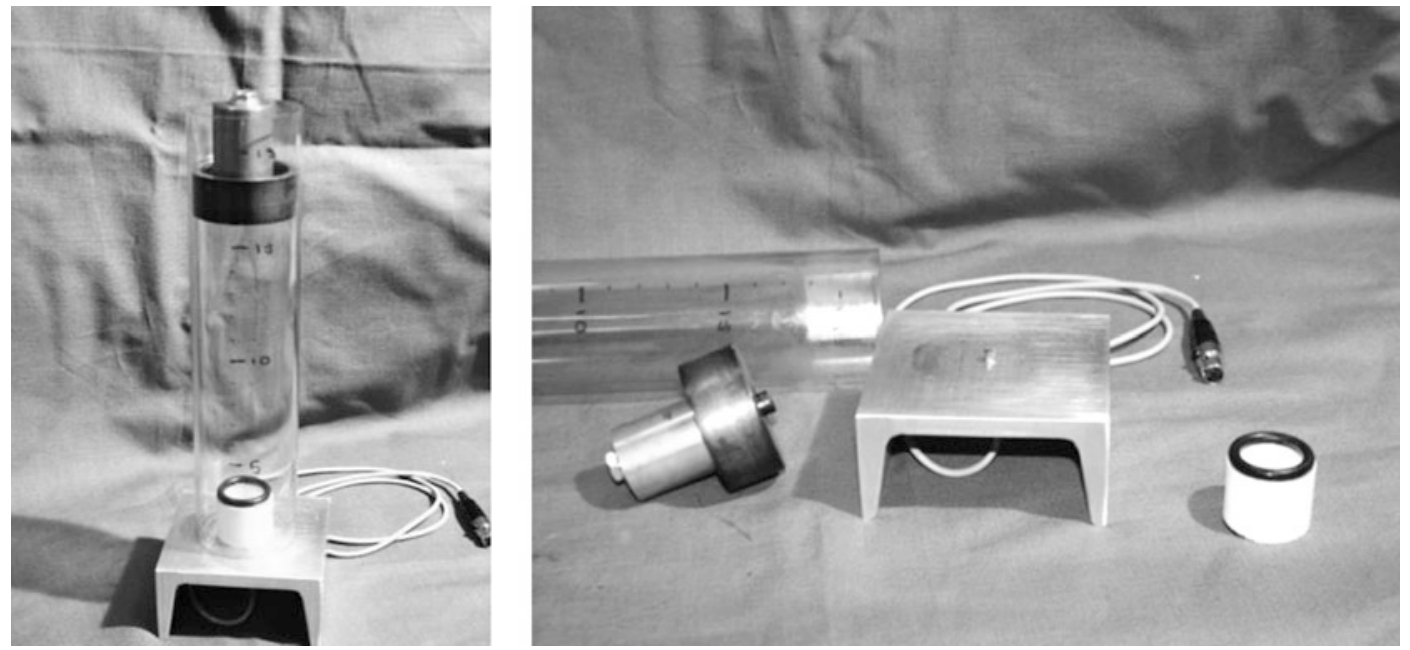

Fig. 7.6 (Left) This simple harmonic oscillator is made from an Airpot ${ }^{\mathrm{TM}}$ Precision Air Dashpot, ${ }^{11}$ consisting of a glass cylinder and a tight-fitting graphite piston of diameter $44.33 \pm 0.03 \mathrm{~mm}$ that acts as a gas spring with stiffness, $\mathrm{K}_{\text {gas }}$. The graphite piston and brass cylinder provide a total piston mass, $m=184.41 \mathrm{gm}$. The bottom end of the glass cylinder is sealed to an aluminum platform with vacuum grease. (Right) An Endevco piezoresistive pressure sensor is threaded into the platform and is protruding through the platform. The microphone's electrical output is connected to a digital storage oscilloscope to allow accurate measurement of the natural period of the freely decaying oscillations that are similar to the trace shown in Fig. 1.18. The small (white) PVC tube with a rubber O-ring on top is placed over the microphone as protection against any possible collision between the microphone and the graphite piston

Repeat part (a), but for pure liquid water, assume its molar mass is $M_{\text {water }}=18.015 \mathrm{gm} / \mathrm{mole}$ and its sound speed is $c=1481 \mathrm{~m} / \mathrm{sec}$ at $20{ }^{\circ} \mathrm{C}$.

2. Ideal Gas Law. The density of air at STP is $\rho_{\text {air }}=1.2923 \mathrm{~kg} / \mathrm{m}^{3}$. What would have to be the pressure of helium gas $\left(M_{H e}=4.0026 \times 10^{-3} \mathrm{~kg} / \mathrm{mol}\right)$ to have the same density at standard temperature? 
3. Loudspeaker cone equivalent piston area. Shown in Fig. 7.5 (Left) is a photograph of a $15^{\prime \prime}$ (nominal) loudspeaker cone connected to a load cell (force gauge) and a supply of pressurized gas. Within the cone-clamping fixture at the bottom of the photo is a pressure gauge. The graph in Fig. 7.5 (Right) shows the pressure difference across the cone vs. the force the cone applied through the load cell. The straight line is a fit to the 12 data points. Based on the graph, determine the effective area of the cone. Report your result in square centimeters $\left(\mathrm{cm}^{2}\right)$. Based on the square of the correlation coefficient for that fit $\left(R^{2}=0.99985\right)$, estimate the uncertainty of your determination of the loudspeaker's effective area.

4. Rüchardt's method. [12] A simple harmonic oscillator that combines a gas spring and a (nearly) frictionless piston (Airpot ${ }^{\circledR}$ ) is shown in Fig. 7.6. The glass cylinder that contains the piston is sealed to a platform that contains a microphone that can measure and record the oscillating pressure within the cylinder if the piston is displaced from its equilibrium position and allowed to execute simple harmonic motion with frequency, $\omega_{o}=2 \pi f_{o}=\left(\mathrm{K}_{\text {gas }} / \mathrm{m}\right)^{1 / 2}$.

The spring constant, $\mathrm{K}_{\text {gas }}$, of a "gas spring" comprised of a cylinder of uniform cross-sectional area, $A$, and volume, $V=A h$, depends upon the mean pressure, $p_{m}$, of the gas contained within the cylinder.

$$
\mathrm{K}_{\text {gas }}=\frac{\gamma p_{m}}{V} A^{2}=\rho_{m} c^{2} \frac{A^{2}}{V}
$$

$A, V=A h$, and $p_{m}$ can be measured accurately. The value of $\omega_{o}$ can be determined from the period of oscillation, $T$.

I used a ruler and a marker pen to place marks separated by $1.0 \mathrm{~cm}$ along the glass cylinder. The microphone and the PVC protector excluded some of the volume within the cylinder. I assumed the compressible volume was determined by the measured height of the piston plus some "fudge" length, $h_{o}$, that would take care of any excluded volumes and any offset in my marking of the cylinder's length. The value of the polytropic coefficient of air, $\gamma_{\text {air }}$, could then be determined by a least-squares fit

Table 7.1 Oscillation periods, $T_{i}$, in milliseconds, for various equilibrium heights, $h_{i}$, of the bottom of the graphite piston measured using the apparatus shown in Fig. 7.6

\begin{tabular}{l|l}
\hline Heights $\left(h_{i}\right)$ & Periods $\left(T_{i}\right)$ \\
\hline$(\mathrm{m})$ & (milliseconds) \\
\hline 0.15 & 70.480 \\
\hline 0.13 & 65.400 \\
\hline 0.19 & 79.725 \\
\hline 0.18 & 77.600 \\
\hline 0.15 & 70.567 \\
\hline 0.14 & 68.143 \\
\hline 0.13 & 65.680 \\
\hline 0.11 & 60.467 \\
\hline 0.10 & 57.880 \\
\hline 0.08 & 50.450 \\
\hline 0.19 & 79.800 \\
\hline 0.18 & 77.867 \\
\hline 0.17 & 75.267 \\
\hline 0.16 & 73.200 \\
\hline 0.14 & 70.850 \\
\hline & 68.467 \\
\hline
\end{tabular}


of the square of the measured periods, $T_{i}^{2}$, plotted against the equilibrium heights of the piston, $h_{i}$ (see Sect. 1.9.3), based on the marks on the cylinder.

$$
\left[\frac{p_{m} A}{4 \pi^{2} m}\right] T_{i}^{2}=\frac{1}{\gamma_{\text {air }}}\left(h_{i}+h_{o}\right)
$$

The polytropic coefficient would therefore be equal to the reciprocal of the slope, $\gamma_{\text {air }}=(\text { slope })^{-1}$, and the "height offset," $h_{o}$, would be the ratio of the intercept to the slope, $h_{o}=$ (intercept)/(slope).

The data in Table 7.1 was acquired at an atmospheric pressure of $97.3 \mathrm{kPa}$. (Is that the mean pressure, $p_{m}$, within the volume?) Using your favorite plotting package, determine $\gamma_{\text {air }}$ and the relative uncertainty in slope of the best-fit line (see Sect. 1.9.2).

The accepted value for the polytropic coefficient of dry air is $\gamma_{\text {air }}=1.403$. What is the relative difference between your experimental value of $\gamma_{\text {air }}$ and the accepted value? If that relative difference is larger than the relative uncertainty in the slope, suggest at least one source of systematic error that could be responsible for that discrepancy.

\section{References}

1. A. Swaminathan, S.L. Garrett, M.E. Poese, R.W.M. Smith, Dynamic stabilization of the Rayleigh-Bénard instability by acceleration modulation. J. Acoust. Soc. Am. 144(4), 2334-2343 (2018)

2. U. S. Department of Labor, Occupational Safety \& Health Administration, Standards - 29 CFR, Standard No. 1910.95(b)(1), Table G-16 - Permissible Noise Exposure

3. U. S. Standard Atmosphere, 1976 (National Oceanic and Atmospheric Administration, Report S/T $76-1562$ (1976)

4. R.P. Feynman, R.B. Leighton, M. Sands, The Feynman Lectures on Physics, vol I (Addison-Wesley, Reading, 1963)

5. P.J. Mohr, D.B. Newell, B.N. Taylor, E. Tiesinga, Data and analysis for the CODATA 2017 special fundamental constants adjustment. Metrologia 55, 125-146 (2017)

6. S.W. Angrist, L.G. Helper, G. Loren, Order and Chaos - Laws of Energy and Entropy (Basic Books, New York, 1967).pg. 215

7. L. Pitre, F. Sparasci, L. Risegari, C. Guianvarc'h, C. Martin, M.E. Himbert, M.D. Plimmer, A. Allard, B. Marty, P.A. Giuliano Albo, B. Gao, M.R. Moldover, J.B. Mehl, New measurement of the Boltzmann constant $k$ by acoustic thermometry of helium-4 gas. Metrologia 54, 856-873 (2017)

8. R. Resnick, D. Halliday, Physics-Part I (Wiley, New York, 1966)

9. L.D. Landau, E.M. Lifshitz, Fluid Mechanics, 2nd edn. (Butterworth Heinemann, Oxford, 1987); ISBN 0-75062767-0

10. D.A. Danielson, Vectors and Tensors in Engineering and Physics (Addison-Wesley, Redwood City, 1992); ISBN 0-201-52426-0

11. G.W. Swift, Thermoacoustics: A Unifying Perspective for Some Engines and Refrigerators, 2nd edn. (ASA Press/ Springer, Cham, 2017), See Sect. 2.2.4; ISBN 978-3-319-66932

12. E. Rüchardt, Eine einfache methode zur bestimmung von $C_{p} / C_{v}$. Phys. Z. 30, 58-59 (1929)

Open Access This chapter is licensed under the terms of the Creative Commons Attribution 4.0 International License (http://creativecommons.org/licenses/by/4.0/), which permits use, sharing, adaptation, distribution and reproduction in any medium or format, as long as you give appropriate credit to the original author(s) and the source, provide a link to the Creative Commons license and indicate if changes were made.

The images or other third party material in this chapter are included in the chapter's Creative Commons license, unless indicated otherwise in a credit line to the material. If material is not included in the chapter's Creative Commons license and your intended use is not permitted by statutory regulation or exceeds the permitted use, you will need to obtain permission directly from the copyright holder. 